\title{
La disfunción sexual eréctil se relacionó más con el uso de medicamentos con enfermedades cardiovaculares
}

\author{
Erectile dysfunction is associated with drug exposures, but less with cardiovascular conditions
}

Francis M, et al. J Urol 2007 Aug 178; 591-596.

\section{Objetivo}

Evaluar la asociación de la disfunción sexual eréctil completa con la presencia de patologías médicas y la exposición a drogas.

\section{Diseño}

Estudio de corte transversal de base poblacional.

\section{Lugar \\ EE.UU.}

\section{Medición de resultados principales}

Hombres mayores de 40 años que respondieron la Encuesta del Exámen Nacional de Salud y Nutrición (NHANES) entre 2000 y 2001. Fueron interrogados por la presencia de disfunción sexual eréctil completa (DSEC) y su relación con síntomas urinarios bajos, enfermedad cardiovascular, diabetes, hipertensión arterial, uso de medicación antihipertensiva selectiva (basicamente betabloqueantes y diuréticos tiazídicos) utilización de antidepresivos, abuso de tabaco y alcohol.

\section{Resultados principales}

De los 1370 hombres encuestados con una media de edad de 54,4 años, $8 \%$ reportó padecer DSEC (IC95\% 6 a 10,2\%).
Tabla 1: asociación entre la presencia de disfunción sexual eréctil completa con otras condiciones médicas y/o con el consumo de fármacos.

\begin{tabular}{|c|c|c|}
\hline & OR ajustado IC95\% \\
\hline \multicolumn{2}{|c|}{ Síntomas urinarios obstructivos } & $2,02(1,2$ a 3,4$)$ \\
\hline \multirow{2}{*}{$\begin{array}{l}\text { Hipertensión } \\
\text { tratada }\end{array}$} & con betabloqueantes y/o diuréticos & $3,07(1,6$ a 5,9$)$ \\
\hline & con otras drogas & $1,33(0,7$ a 2,6$)$ \\
\hline \multicolumn{2}{|l|}{ Diabetes } & $2,57(1,3$ a 5,2$)$ \\
\hline \multicolumn{2}{|c|}{ Uso de antidepresivos } & $5,15(1,7$ a 15,9$)$ \\
\hline \multicolumn{2}{|l|}{ Tabaquismo } & $1,93(1,2$ a 3,2$)$ \\
\hline \multicolumn{2}{|c|}{ Enfermedad cardiovascular } & $0,85(0,4$ a 1,8$)$ \\
\hline \multicolumn{2}{|c|}{ Consumo de más de siete tragos semanales de alcohol } & $0,52(0,3$ a 1,1$)$ \\
\hline
\end{tabular}

\section{Conclusiones}

La presencia de síntomas urinarios obstructivos, diabetes, hipertensióna arterial tratada con fármacos selectivos y el uso de antidepresivos estuvo asociada en forma independiente a padecer DSEC. Los médicos deberían considerar el impacto de estas medicaciones y otras condiciones comórbidas al evaluar pacientes con disfunción sexual.

Palabras clave: disfunción sexual eréctil, fármacos, comorbilidades.

Key words: sexual erectil disfunction, drugs, medical conditions.

Fuente de financiamiento: National Center for Health Statistics (EEUU).

\section{Comentario}

Podríamos decir que hay un antes y un después del descubrimiento del sildenafil en cuanto al manejo clínico de la disfunción sexual eréctil (DSE). Antes, este tipo de artículos que intentan demostrar qué fármacos y qué entidades comórbidas se asocian con un mayor riesgo de tener DSE eran bienvenidos por la comunidad médica y quizá influían en las conductas clínicas en el consultorio. Hoy en día, sin embargo, es probable que la opción más sencilla para ayudar a mejorar la función eréctil a los pacientes con depresión que usan antidepresivos y a los pacientes con diabetes y/o hipertensión medicados con diuréticos y/o betabloqueantes, sea ofrecerles sildenafil para mejorar su DSE con el objetivo de poder conservar el tratamiento de la condición clínica que motivó la administración de los fármacos supuestamente implicados en la DSE'1.

No obstante esta introducción, no deja de ser interesante que se sigan publicando artículos basados en datos epidemiológicos que no hacen más que reforzar conocimientos que tenemos hace muchos años respecto a que las personas mencionadas arriba sufren más DSE que la población general. Más allá de hacer un llamado a la prevención primaria y de optimizar el tratamiento en los individuos que sufren hipertensión arterial y diabetes intentando minimizar sus efectos adversos de acuerdo a las preferencias de los pacientes, una reflexión que podría hacerse es que los médicos deberíamos indagar especialmente acerca de la función sexual en estos grupos.

Por último, hay un dato que es característico de este artículo y que difiere de lo que se viene publicando en la bibliografía especializada: los pacientes con enfermedad cardiovascular establecida no tuvieron una prevalencia muy elevada de DSE. Esta información llama la atención ya que en la mayoría de las series publicadas previamente ${ }^{2}$ este grupo había sido identificado como más vulnerable.

\section{Conclusiones del comentador}

La DSE es una entidad muy prevalente. Es importante que los médicos interroguemos a nuestros pacientes acerca de la función sexual y que no esperemos a que nos consulten. Este artículo nos indica que los grupos más expuestos son aquellos que toman antidepresivos, así como los medicados con betabloqueantes y/o diuréticos por hipertensión y/o diabetes.

Esteban Rubinstein [ Servicio de Medicina Familiar y Comunitaria. Hospital Italiano de Buenos Aires. esteban.rubinstein @hospitalitaliano.org.ar ]

Rubinstein E. La disfunción sexual eréctil se relacionó más con el uso de medicamentos con enfermedades cardiovaculares. Evid. actual. práct. ambul; 11(1): 14, Ene-Feb.2008. Comentado de: Francis M, Kusek J, Nyberg L, et al. The contribution of common medical conditions and drug exposures to erectile dysfunction in adult males. J Urol 2007 Aug 178; 591-596. PMID 17570434

\section{Bibliografía}

1. Gueglio, Guillermo; Rubinstein, Esteban; Carrete, Paula. Disfunción eréctil. Buenos Aires: delhospital ediciones, 2006.

2. Kloner RA, et al. Erectile dysfunction in the cardiac patient: how common and should we treat? J. Urol 2003; 170 (2 pt 2): S46-50; discussion S50. 